\title{
Timbre, Genre, and Polystylism in Sonic the Hedgehog 3
}

\author{
Megan Lavengood
}

Sonic the Hedgehog 3, a video game released in 1994, is a classic "platformer" video game. Sonic the Hedgehog was Sega's flagship series for its new home console, the Sega Genesis, functioning essentially as their version of Mario, but blue and gritty. ${ }^{1}$ The manual for the original Sonic the Hedgehog video game introduces Sonic to the world: "Only one tough dude can put an end to the demented scientist's [Dr. Robotnik's] fiendish scheme. It's Sonic, the real cool hedgehog with the spiked haircut and power sneakers that give him super speed" (Sega 1991, 4). Sonic is not at all like an Italian plumber in a mushroom kingdom-he's positioned through Sega's marketing materials as a rebellious and modern fighter, sporting a trendy haircut and sneakers.

Like many video games of the late 1980s and early 1990s, Sonic the Hedgehog 3 (hereafter Sonic 3) is structured around the player traveling through many levels, each with a different setting; the music reflects these setting changes-a volcano, a forest, a city, a lake, and so on-by invoking a variety of styles. These various locations, although distinct, do not result

${ }^{1}$ Mario is the main character of Nintendo's flagship series, Super Mario Bros.

M. Lavengood $(\bowtie)$

City University of New York Graduate Centre, New York City, NY, USA

(C) The Author(s) 2019

N. Braae, K. A. Hansen (eds.), On Popular Music and Its Unruly

Entanglements, Pop Music, Culture and Identity, https://doi.org/10.1007/978-3-030-18099-7_11 
in an incoherent gameplay experience, since the varied settings are bound together by the context of the larger storyline and the consistency of collecting coins and fighting Dr. Robotnik in each level. Far-flung locales and their corresponding music contrast this consistency in gameplay, bringing excitement and a sense of discovery to the player's experience. Composed by a team of pop songwriters, the styles represented in the Sonic 3 soundtrack include calypso, funk, carnival, new wave, and prog rock. The soundtrack thus does not cohere in terms of a singular musical genre or category; instead the soundtrack is polystylistic and brings forth numerous genre associations. ${ }^{2}$ In short, Sonic 3's soundtrack relies on polystylism to aurally enhance player experience.

Some style/setting connections in Sonic 3 are obvious: the use of the Trinidadian soca style for the tropical setting of the level Angel Island Zone is straightforward; the use of thrash metal for the final boss is likely meant to index a hyper-masculine and fear-inducing atmosphere; the music for Carnival Night Zone borrows the chromatic descending melodic motive from Julius Fučík's march titled "Entrance of the Gladiators" (a tune that is perhaps not well-known by the title, but is certainly known by many as "that circus song that goes like 'doot-doot-doodledoodle-dootdoot-do-do""). ${ }^{3}$ But other connections between a level's setting and the genre referenced through its music are more nuanced. For example, in the level Ice Cap Zone, which is set on a snowy mountain as the player controls Sonic snowboarding down the slopes, rather than relying on typical markers for wintertime (bells, metallic sounds), the music is written in an

\footnotetext{
${ }^{2}$ Throughout this essay, I reference both "genres" as they exist as complex sociological phenomena and "styles" as they are deployed within the Sonic 3 soundtrack as a polystylistic musical text. While this might imply a strict separation between genre and style, my opinion is that the division between these two terms is not so clear-cut. Many scholars believe that styles are defined through musical characteristics (the "music itself"), while genres are sociological; I find the two concepts to be too intertwined to untangle them in any practical context. For a lengthy argument in this vein, see Johnson (2018): "Is it really possible, as [Allan] Moore suggests, that style 'simply brackets out the social or at least regards this realm as minimally determining, where it is considered to operate with a negotiable degree of autonomy' $(2001,441)$ ? How autonomous are stylistic units? Surely style creates chains of signification as well that extend beyond the 'music itself.' The ambiguity and irreducibility inherent in these pseudo-definitions reveals and introduces analytical and conceptual adversities..." (27). I find the genre/style distinction to be quite slippery, although I've attempted to maintain clarity here.

${ }^{3}$ For more on metal's association with the hyper-masculine, see Weinstein (2000) and Wallmark (2014).
} 
early-1980s new wave style. Here, the connection may be understood not as a reference to a physical location, but instead as a cultural reference. In the 1980s and still today, new wave is associated with a cold, harsh, and clean aesthetic. ${ }^{4}$ In cases such as these, where the relation between the music's style and the game's setting is harder to discern, the contributions of timbre and its socio-cultural connotations are foregrounded.

In this essay, I will provide a close analysis of Ice Cap Zone and another level, Marble Garden Zone, and detail how timbre, as a musical parameter separate from instrumentation, can evoke specific intertextual and extramusical associations for a listener. The genres invoked in the soundtracks of each of these levels do not have an immediately obvious relationship to the setting: the wintry Ice Cap Zone uses new wave, while the Grecianstyled Marble Garden Zone uses funk. I analyze how timbre, instrumentation, and other genre signifiers in these levels of Sonic 3 connect to each level's setting and to the overall narrative of the video game, and reveal the complex semiotic meanings generated by these surprising music/setting pairings. In doing this, I will show how timbre, a musical parameter that remains overlooked in a great deal of music analysis, might inform and enhance analyses of narrative, both within video game music and beyond.

Because the object of analysis here is somewhat niche, I will now situate Sonic 3 and the Sonic franchise as a whole within video game culture and provide relevant technical details of the Sega Genesis sound system. The music of the Sonic series was, and remains, celebrated by gamers due to both the high sound quality provided by the Genesis technology as well as the innovative compositions themselves. ${ }^{5}$ The soundtrack of Sonic 3 is especially popular for many reasons, one of which is that many believe Michael Jackson was one of the game's composers. ${ }^{6}$ As recently as 2016, Sega has denied that Jackson had any role in Sonic 3, yet the theory remains widely accepted based on a few key pieces of evidence. ${ }^{7}$ Firstly, there are

\footnotetext{
${ }^{4}$ Cateforis illustrates this through an analysis of the music video accompanying "Video Killed the Radio Star" (2011, 5-9).

${ }^{5}$ As an example of praise for Sonic music, see a discussion on Reddit's video game subreddit about the series' music: https://www.reddit.com/r/Games/comments/lrj0li/ rgames_music_discussion_sonic_the_hedgehog.

${ }^{6}$ See, for example, Lynch (2016), Van Luling (2016), HXC (2005), and the Sonic Retro page "Michael Jackson Involvement with Sonic 3" (2017).

${ }^{7}$ From Van Luling (2016): "Sega maintains it never worked with Jackson on Sonic 3, and is 'not in the position to respond' to questions about allegations to the contrary. 'We have nothing to comment on the case,' the company said."
} 
the clear stylistic symmetries between the Sonic 3 soundtrack and Jackson's oeuvre, and secondly, other Sonic 3 composers have attested to his involvement (Black \& White 2009). As for why Jackson's name is not credited for the compositions, and why Sega continues to insist that Jackson was not involved, fan theories offer two possible explanations: either Jackson quit because the sound quality wasn't good enough, or Sega fired Jackson in reaction to the contemporaneous accusations that Jackson molested a child. ${ }^{8}$

I will not be investigating such theories in this paper, but Jackson's possible involvement is worth noting here because, as David Brackett neatly summarizes in his 2016 book Categorizing Sound, Michael Jackson is a "quintessentially postmodern figure, transcending other, more basic dualisms, such as those based on race, gender, and sexuality" (301). In other words, Michael Jackson himself is a figure that defies generic categorization: as Brackett articulates, his output was notable for its "crossover" hits that blurred the lines between genre categories. Returning to Sonic 3, many of the style choices seem to mirror Jackson's own polystylistic output, most obviously in the pop-, R\&B-, funk- and hip-hop-oriented tracks. But Jackson's possible influence is perceptible also in other genres that one might sooner align with the umbrella genre of art music (Carnival Night Zone is one of the famous locations of supposed Jackson influence). While it remains impossible to identify a conclusive connection between Jackson and Sonic, I see a strong resonance between the two bodies of music.

The Sonic franchise was nearly exclusively released on video game consoles made by Sega (a company that readers may not be acquainted with, since Sega stopped making consoles in 2001). Given the wide success of the Nintendo Entertainment System (NES), an 8-bit home video game console which was released in 1983, it might seem that no other company could compete with Nintendo's dominance of the market, but in 1988, Sega dealt a serious blow to Nintendo by releasing their own 16-bit console, the Sega Genesis. ${ }^{9}$ The Genesis was a huge hit with video game enthusiasts ("gamers") of the time. The 16-bit graphics were far more

\footnotetext{
${ }^{8}$ Van Luling (2016) provides further details: three of the game's composers say that Jackson "was disappointed by how different the music sounded on Sega's console when compressed from that 'high profile' sound to bleeps and bloops," while the executive coordinator of Sonic 3 said it was due to molestation allegations.

${ }^{9}$ The console was released in 1988 under the name Mega Drive in Japan. In 1989, it was released in North America, but under the name Sega Genesis, due to an existing trademark on the term Mega Drive.
} 
detailed than the 8-bit graphics of the NES, and the console also had superior sound to match the advanced graphics. With Nintendo still on their 8-bit NES system, Sega's 16-bit Genesis was a clear threat, and as Karen Collins describes in her book Game Sound: An Introduction to the History, Theory, and Practice of Video Game Music and Sound Design (2008), the console's high-quality audio created much of the appeal of the Genesis. Sega used a Yamaha FM synthesis sound chip, the YM2612, which used technology quite similar to the immensely popular Yamaha DX7 FM synthesizer, a nearly omnipresent sound in popular music in the 1980s. ${ }^{10}$ Like the DX7, the YM2612 excelled not so much in orchestral sounds, but rather in keyboard sounds, guitar sounds, and percussion sounds. Accordingly, the soundtracks for Sega games often took on a hard rock or progressive rock style, capitalizing on the strengths of the YM2612. This use of rock styles distinguished Sega's music from the pop-oriented styles familiar from the 8-bit NES tunes.

Readers may also appreciate a brief overview of the sound chip technology of the Sega Genesis, as I will sometimes reference instruments by their channel. The Yamaha YM2612 FM synthesis chip is the primary sound chip, but the Genesis also has a secondary sound chip, the Texas Instruments SN76489 Programmable Sound Generator (hereafter PSG). The YM2612 has six FM synthesis channels. Each channel has four operators, which can be arranged into seven different algorithms to generate a wide variety of different timbres (Fig. 11.1). ${ }^{11}$ These channels are monophonic - they can only sound one note at a time. The secondary sound chip, the SN76489 PSG, has four channels: three channels use digital oscillators to generate square waves, while the fourth is a white noise generator, useful for percussion sounds and other noise-based sound effects.

To analyze timbre in the soundtrack of Sonic 3, one tool I use is spectrogram analysis. A spectrogram approximates the sound waves present in a signal with a visual representation that is easy for our brains to process out-of-time. This represents the principle advantage of consulting spectrograms: they visually represent timbre in a way that is akin to how the notated musical score represents other domains of music analysis. A spectrogram charts a sound signal, showing frequency on the $y$-axis and time on the $x$-axis, while showing amplitude with changes in color, thus

\footnotetext{
${ }^{10}$ For more discussion of the significance of the DX7 in 1980s music, see Lavengood (2019).

${ }^{11}$ This is succinctly discussed in Collins $(2008,40-42)$.
} 
Eight algorithmic configurations of the FM chip's operators, from the Sega Genesis Technical Manual. Slots are indicated by shading.

\begin{tabular}{|c|c|c|}
\hline Algorithm \# & Layout & Suggested uses \\
\hline 0 & $1-2-3-4 \longrightarrow$ & Distorted guitar, "high hat chopper," bass \\
\hline 1 & $\begin{array}{l}1 \\
2\end{array}$ & Harp, "PSG"-like sound \\
\hline 2 & $1-3$ & Bass guitar, electric guitar, brass, piano, woodwinds \\
\hline 3 & $\underset{4}{1+2}$ & Strings, folk guitar, chimes \\
\hline 4 & $\underset{3-4}{-1-2]}$ & Flute, bells, chorus, bass drum, snare drum, tom-tom \\
\hline 5 & 1 & Brass, organ \\
\hline 6 & $\begin{array}{r}1+2 \\
3 \\
4\end{array} \rightarrow$ & $\begin{array}{l}\text { Xylophone, tom-tom, organ, vibraphone, snare } \\
\text { drum, bass drum }\end{array}$ \\
\hline 7 & $\begin{array}{l}1 \\
2 \\
2 \\
3 \\
4\end{array} \rightarrow$ & Pipe organ \\
\hline
\end{tabular}

Fig. 11.1 Table 3.1 from Collins $(2008,41)$

visualizing the relative weighting of the energy distribution along a given frequency range and providing a visual representation of most of the elements that define a timbre. In layperson's terms, a spectrogram shows the volume of all the frequencies present in a sound signal, and the way those volumes and frequencies change through time. This means that a spectrogram shows not only the fundamental pitch, but also all the overtones that the ear combines into a single tone with a unique timbre. This visually illustrates many components of the complex phenomenon that listeners perceive as "timbre." 
I create the audio signals for my spectrogram analyses with a program called Audio Overload, a freeware program created by Richard Bannister that "emulates the sound hardware of vintage consoles and computers, allowing you to listen to completely authentic renditions of classic video game tunes" (2016). Audio Overload plays files in .vgm format (among others). These .vgm files are created by video game music enthusiasts, through unofficial, often open-source software. ${ }^{12}$ Sega Genesis emulators, which are software designed to make a computer function like a Sega Genesis console, sometimes permit "logging" of the signals that create the music. Through the logging process, a . vgm file is created. In other words, the signals themselves are what is recorded, not the audio-a distinction that is somewhat similar to recording data via MIDI versus recording audio with a microphone and a digital interface. In the .vgm file, separate signals are logged for each of the YM2612's six FM channels, as well as the four channels on the SN76489 PSG. Audio Overload can read that data and translate it into authentic Sega Genesis audio, without needing a hardware Sega Genesis on hand. Audio Overload does not export to .wav or other audio formats, but more software can be used to record the audio output. The benefit for the analyst of using Audio Overload instead of a real console is that Audio Overload allows each of the soundtrack's six channels of sound to be turned on and off as needed. When I analyze the timbre of the "instruments" used in these tracks, I put each channel on solo to record it in isolation. When I create a spectrogram from a single channel, there is much less interference than when I generate a spectrogram from all the channels mixed together. Figure 11.2 demonstrates this neatly: 2 a uses only one channel as its signal, whereas $2 b$ shows all 10 channels together.

Timbre can be appraised by a listener in a fraction of a second, rendering it one of the most immediately perceptible elements of musical experience. ${ }^{13}$ Many have noted the significant relationship that timbre and

\footnotetext{
${ }^{12}$ This is why many of the resources in my bibliography are not scholarly sources, and frequently have no author, or an author going by a pseudonym —all of this work occurs online, via forums and chat rooms.

${ }^{13}$ By this, I simply mean that listeners have instantaneous judgments of and reactions to timbres. Consider how timbre lets you know whose voice is on the other end of your phone call, and allows you to separate familiar sounds from unfamiliar sounds (like discerning a normal whirring in your car's air conditioning from a new screeching sound that suddenly starts sounding when you apply the brakes).
} 

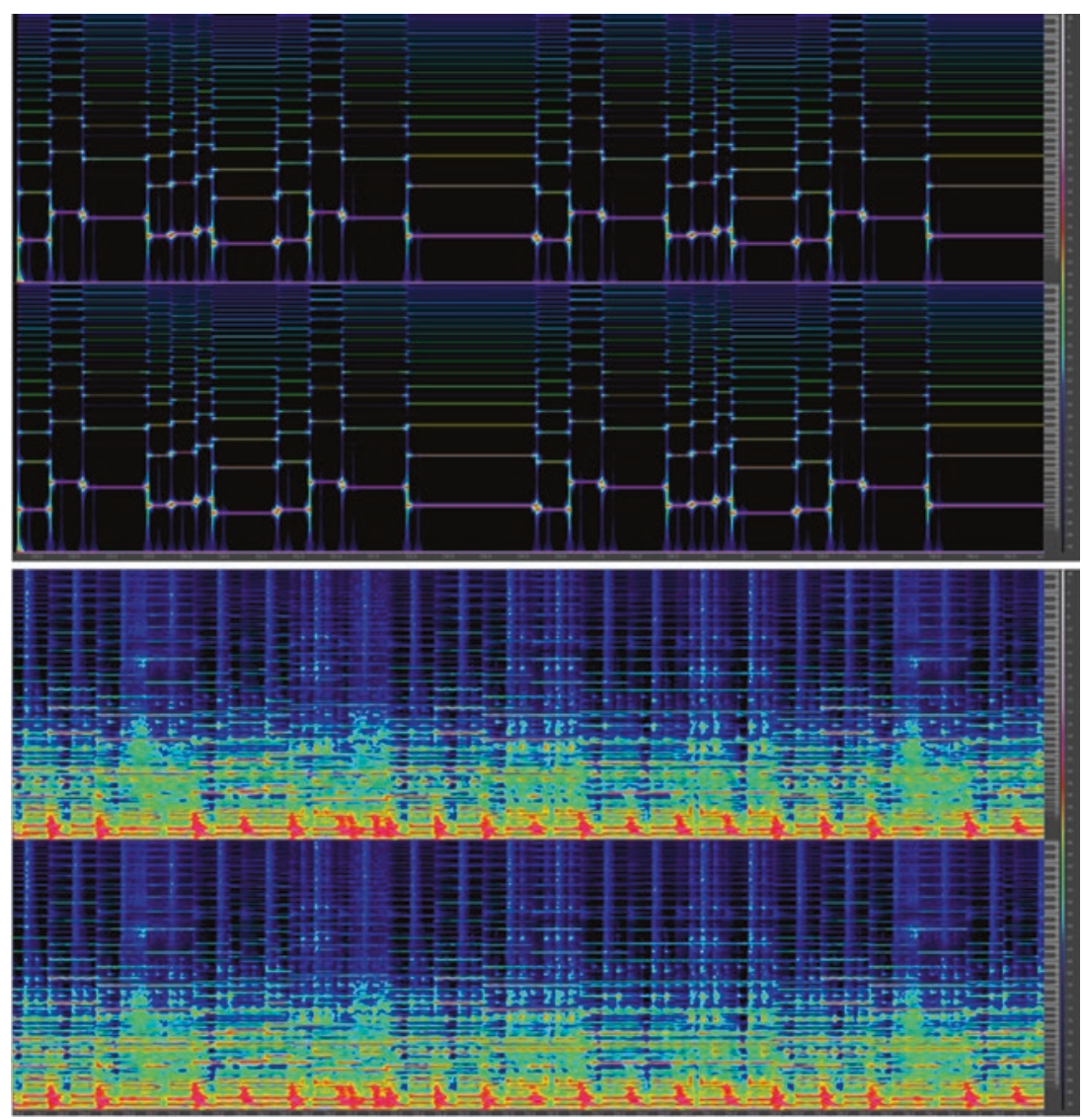

Fig. 11.2 A spectrogram of a signal from a single channel (a) versus a signal from ten channels (b)

instrumentation have in shaping perceptions of genre, and thus it is perhaps no surprise that categorizing music into an appropriate genre is similarly instantaneous: a recent study found that listeners can accurately assess genre within one quarter of one second. ${ }^{14}$ Despite the proliferation

${ }^{14}$ Gjerdingen and Perrott state "[Timbral] information can be highly indicative of particular genres. ... The results of this study suggest that a highly reduced combination of melodic, bass, harmonic, and rhythmic features can help to classify genre if these features are coupled with an accurate acoustic signal" $(2008,98)$. David Blake, speaking specifically about indie 
of rhetoric, especially from composers and artists, stating that genres are artificial, genre remains a powerful tool for music categorization and, perhaps more importantly, for describing the intertextual associations that listeners make when they hear a given set of timbres (for example, the association of distorted electric guitar with the heavy metal genre, or the association of gangsta rap or g-funk backing tracks with a high-pitched sine wave with portamento). ${ }^{15}$ Scholars such as Brackett have demonstrated that genres are inherently intertextual and evocative of a set of societally recognized meanings. ${ }^{16}$ If a genre conjures relations to an entire musical aesthetic in the mind of a listener, a polystylistic musical text could on the surface seem senseless or bizarre, like randomness for randomness's sake, as opposed to an iteration of a coherent aesthetic or an artistic agenda. Yet polystylistic music instead generates new meanings of its own that rely upon those stylistic intertextualities and genre associations. ${ }^{17}$

I consider the soundtrack of Sonic 3 to be a polystylistic musical object. Polystylism has a long history in both art and popular music. Focusing on the latter, polystylism in popular music is frequently tied to studies of sampling in hip-hop and other genres, as samples that originate from many different genres are often used freely alongside one another. Sampling

music, states “...timbre, more than any other musical parameter, expresses extramusical differentiation in independent music genres. Timbral choices ...connect with discourses of differentiation on cultural, political, economic, and/or generic planes" (2012). Zachary Wallmark echoes these thoughts: “... musical ordering-based to a significant degree on rapid perceptual differences in timbre-is closely connected to social ordering. Understanding timbre's role in this dynamic is thus crucial, I maintain, for future research in the sociology, ethics, and social psychology of music" $(2014,264)$. Gjerdingen and Perrott also provide the quarter-of-a-second measurement for timbre perception $(2008,97)$.

${ }^{15}$ Brackett describes this "genre is dead" stereotype thusly: “...musicians and consumers often resist requests to categorize themselves, insisting that their tastes are unclassifiable. It is common to hear discussions that have invoked the idea of genre end with the declaration that musical genres do not really exist, that they are mere fabrications of the music industry" $(2016,1)$.

${ }^{16}$ Brackett's entire book (2016) deals with this concept: how genres seem quite difficult to pin down, precisely because they seem to network in many different ways, and are resistant to neat divisions or branching structures. He summarizes: "Put another way, genres are not static groupings of empirically verifiable musical characteristics, but rather associations of texts whose criteria of similarity may vary according to the uses to which the genre labels are put. 'Similar' elements include more than musical-style features, and groupings often hinge on elements of nation, class, race, gender, sexuality, and so on" (3-4).

${ }^{17}$ Authors such as Rebecca Leydon (2010) and Christine Boone (2018) have discussed this issue in experimental rock music and in mashups, respectively. 
began with DJs quickly changing out vinyl records on their turntables; in the digital age, sampling quickly took over many forms of music-making. ${ }^{18}$ Sampling is most evident in hip-hop and its related genres, but some authors have argued that sampling, as an aesthetic, has influenced even music that is entirely newly composed: in her essay "Recombinant Style Topics," Rebecca Leydon theorizes that digital-era polystylism can be understood as "second-era sampling," where even if a polystylistic musical text doesn't use samples at all, using eclectic and unreconciled genres in a newly-composed piece of music may be understood as influenced by the aesthetic of sampling (2010). An analysis from this viewpoint demonstrates the drawing together of seemingly disparate pieces into an organic whole: a whole that is paradoxically bound together through these disparities.

I view the implementations of styles and their attendant genre traits in Sonic 3 as amalgams of music-analytical ideas, which I have termed "genretopics." Musical genres, styles, topics are closely linked, as evidenced in Mirka's statement that "it is their deployment in other pieces and mixtures with other styles that turns styles into topics" $(2014,1)$. Topics generate musical meaning by creating a web of intertextual references with other instantiations of the topic (Johnson 2017). ${ }^{19}$ Topic theory was first applied to music of the eighteenth century, because, as demonstrated by Ratner in Classic Music: Expression, Form, and Style (1980), topics actually would have been recognized as such by an educated eighteenth-century listener. Mirka notes that topics thus "allow one to gain access to [the meaning and expression of eighteenth-century music] in a way that can be intersubjectively verified" $(2014,1)$. Much like an eighteenth-century listener would be fluent enough in contemporaneous musical styles to identify the meaning behind a march, a hunt, or a Turkish topic, Sonic 3's players in the 1980s and 1990s would similarly be fluent enough in various popular genres to recognize the deployment of typical musical traits of a genre like new wave or funk, and each genre's own web of meanings and social connotations. In an age of musical omnivorousness, composers can rely on the assumption that the listening audience will be fluent enough in many diverse genres to appreciate the differences between them, thereby allow-

\footnotetext{
${ }^{18}$ For a thorough history of sampling and other forms of borrowing as it applies particularly to hip hop culture, see Schloss (2004) and Williams (2013).

${ }^{19}$ See Johnson (2018) for a thorough demonstration of this principle, i.e., the semiotic code.
} 
ing composers and listeners to work together to generate musical meaning through intertextual, polystylistic references. The genre-topics discussed in this paper, however, are not defined in the same manner as Ratner's Classical topics. While topics in eighteenth-century music - the march, the hunt-are principally defined through melodic, harmonic, and rhythmic content, in Sonic 3, topics are conveyed primarily through instrumentation and timbre. This is similar to Leydon's use of styles as topics in analyzing polystylistic popular music (2010). Leydon notes that for popular music, it is instrumentation which "bears the bulk of the semiotic burden" when style is communicated to the listener:

In popular music, however, it is not unusual for single instruments to serve as tokens for style - the banjo standing in for "bluegrass" or the Roland transistor bass for "House," for instance-and it may be one of its key features that genre and instrumentation are so closely connected. ... Consequently, instrumentation serves as a reliable and sufficient index for rock's historical styles $(2010,209)$.

Leydon here stops short of detailing specifically how instrumental timbres might signify these different styles; it seems unnecessary in these contexts to work to justify that the Clavinet sound would be associated with Stevie Wonder, for example, and thus with a funk genre. The equivalencing between Clavinet and funk is an example of a principle of semiotics and topic theory in music that Philip Tagg (2012) terms a "genre synecdoche." Essentially, genre synecdoches are signifying elements of a genre that call up the meaning of the entire genre through the deployment of just that single element. Tagg says of genre synecdoches:

Put tersely, genre synecdoches contain three stages of semiosis in a connotative chain: [1] from certain sounds considered, rightly or wrongly, as typical for a "foreign" musical style to the totality of that same style; [2] from that style to the genre of which it's considered to be part; [3] from that genre to the rest of the culture of which that "foreign" style and its concomitant genre are thought to be part (525).

Sonic 3 problematizes the genre synecdoche equation, as Sonic 3 does not use a Clavinet to signal funk, and in fact, it does not use any other "real" instrument: all sounds are approximated, synthesized, through the YM2612 FM digital sound chip. Simply equivalencing certain instruments, i.e., the instrumentation of a track, as being indicators of certain 
genres does not help identify genre-topics when all the instruments are the same (the YM2612). The comparatively blunt tool of instrumentation, on its own, is not sufficient; study must instead turn to the finer details of timbre and how those timbres represent various genres.

Spectrogram analysis is only the first step in the timbre analysis process, however. Human beings do not perceive timbre in the same way as a computer, because we attune not only to acoustic data, but also to nonacoustic associations we make with the timbres-intertextual relationships between timbres and other phenomena. Thus, in order to reveal deeper meanings communicated by timbres, I discuss socio-cultural implications that pair with each of these timbral attributes and the role of timbre in the creation of genre-topics. Through timbre, I demonstrate interactions between timbres, genres, and larger contexts, and how deployment of genres throughout Sonic 3 reveal aspects of its contemporaneous culture.

\section{ICE CAP Zone}

The soundtrack for Ice Cap Zone alternates A and B sections. The sections are differentiated primarily by the lack of melody in A sections-the A sections sound somewhat introductory in this way. For this reason, I focus on the thicker-textured B sections of Ice Cap Zone's music. To summarize, the instruments might be grouped into percussive sounds, middle voices, bass line, and melody. The drum sounds are split between PSG channel 4, which creates the hi-hat sound, and YM channel 6, which combines bass drum, snare, and a few more unorthodox percussive sounds, such as a record scratch, a simulation of someone shouting "hey!," and an orchestra hit. The bass is in YM channel 1. YM channels 3, 4, and 5 provide middle voices which fill out the harmonies; channel 2 also functions as a middle voice in the A section. In the B sections, however, YM channel 2 combines forces with PSG channels 1 and 2 to create a thick, strong, synth lead sound.

Ice Cap Zone is accompanied by new wave-style music, rather than a more typical wintry-themed soundtrack. New wave is a genre that is notoriously hard to define-Theo Cateforis dedicates an entire book (2011) to the interrogation of the meaning of "new wave" - but to summarize it briefly, two defining aspects of new wave emerge: (1) a modern aesthetic and (2) the influence of disco and punk and the synthesis of their musical features. Cateforis focuses on the preoccupation of new wave artists with 
the notion of "modernity," a term which might have different meanings to different artists, but which he nevertheless finds to have ubiquitous influence on both musical and social aspects of new wave. In terms of musical features, Cateforis demonstrates that new wave is influenced by both disco and punk. From punk, new wave adopts an aesthetic of rebelliousness via amateurism, represented musically through simplistic performing techniques such as reliance on arpeggiators and pre-recorded sequences, defying those that would instead place musical value on virtuosity. From the disco genre comes the groove-based compositional process, often involving danceable rhythms, drum machines, syncopated and funk-influenced bass lines, and synthesizers, which results in a layered or heterogenous musical texture (as opposed to the homophonic texture more typically associated with other pop genres). Well-known songs that could serve as an aural point of reference for new wave as a genre might be "Don't You Want Me" by The Human League (1981), "Cars" by Gary Numan (1979), or "Video Killed the Radio Star" by The Buggles (1979).

Ice Cap Zone's musical features align especially strongly with the discobased new wave markers, as well as the aesthetic of modernity. In Ice Cap Zone, four-to-the-floor bass drum, driving rhythms in the bass line and in upper synthesizers, and a layered approach to musical texture make this track instantly recognizable as emulating new wave music. In fact, the main composer of this music, Brad Buxer, was in a new wave band called The Jetzons, and he based this zone's music on one of the band's tracks, titled "Hard Times." Listening to "Hard Times" reveals that the instruments used in Ice Cap Zone are also meant to evoke the new wave genre. While the use of synthesizer in any Sega Genesis game is trivial-all sounds are synthesized-all sounds in "Hard Times" also are synthesized. In other words, the timbres used in Ice Cap Zone are meant to sound like synthesizers, instead of sounding like imitations of instruments. Because of their cultural connection to modernity and the future, synthesizers are a hallmark of new wave (Cateforis 2011, 151).

New wave is also characterized timbrally as sounding "clean" or "bright," timbral analogies that are often contrasted with timbral "warmth." Part of the reason for this association with brightness and cleanness results from new wave's use of FM digital synthesizers, like the Yamaha DX7 (which uses the same synthesis technology as the YM2612). ${ }^{20}$

\footnotetext{
${ }^{20}$ This association between cold/harsh/clean and the Yamaha DX7 is discussed further in Lavengood (2019).
} 
FM synthesizers like the DX7 are often accused of lacking a "warm" sound. For example, Richard Cottle, a keyboardist and film composer, said in 1986 that "The one thing that the DX can't seem to deliver is a good warm sound" (qtd. in Elen 1986, 38-39). Or consider the words of Bob Moog, inventor of the Moog synthesizer:

In subjective terms, the quality of fluorescent light is harsh compared to the warm light of an incandescent bulb or sunlight, and this is because of the way the light inside of a fluorescent bulb is made compared to how the light inside an incandescent bulb is produced. They're two different physical processes, just the same way as the process of digital sound generation is different from analog sound generation. (Qtd. in Vail 2005, 102)

Moog is implying here that digital production is correspondent to fluorescent light, and analog production to incandescent light, and that therefore digital sound is harsh and not-warm. In short, because musicians and listeners like Cottle and Moog categorize the new wave sound as being not-warm, an association with coldness can arise in turn. Scrutinizing the timbres used in the backing track for Ice Cap Zone reveals how this cold, new wave sound is produced.

The bass sound is particularly indicative of a new wave style. In new wave music, the bass is often not played by an actual bass guitar, but rather by a synthesizer. ${ }^{21}$ This allows for rapid attacks that would be difficult to execute on a real bass guitar, which becomes a hallmark of bass lines in new wave music. In this sense, Ice Cap Zone features a typically new wave bass line, as it is filled with sixteenth note rhythms even while maintaining a tempo of $138 \mathrm{bpm} .{ }^{22}$ On top of having stylistic rhythms, Ice Cap Zone uses a bass timbre that resembles another popular digitally-synthesized bass sound from the Yamaha DX7. Figure 11.3a is the spectrogram for the YM channel 1, the bass channel, as it sounds in the Bl section; $11.3 \mathrm{~b}$ is the spectrogram for a Yamaha DX7 BASS 1 preset. Both these sounds emulate a slap bass sound, which was typical of disco and funk music and thus was eventually imported into new wave. In terms of specific timbral features shown in the spectrogram, both sounds have a very bright attack,

${ }^{21}$ This is part of a larger trend in new wave to eschew not just bass guitars, but all guitars, from the group, in favor of synthesizers, which seem more "modern" (Cateforis 2011, 151-54).

${ }^{22}$ To view transcriptions, navigate to the author's personal website: www.meganlavengood.com/sonic. 
seen in the tall, vertical stripes sent up from the initiation of each tone. Both sounds have a strong second partial, louder than the fundamental, which can be seen by comparing the bottom horizontal stripe, the fundamental, to the stripe above it; the lighter pink color indicates greater amplitude, as shown by the scale on the right of the spectrogram. While not identical, the resemblances between the two sounds are clear, visually and aurally. Because the DX7 BASS I preset was so prevalent in new wave music (Lavengood 2019), the bass timbre used in Ice Cap Zone further evokes the new wave genre (Fig. 11.3).
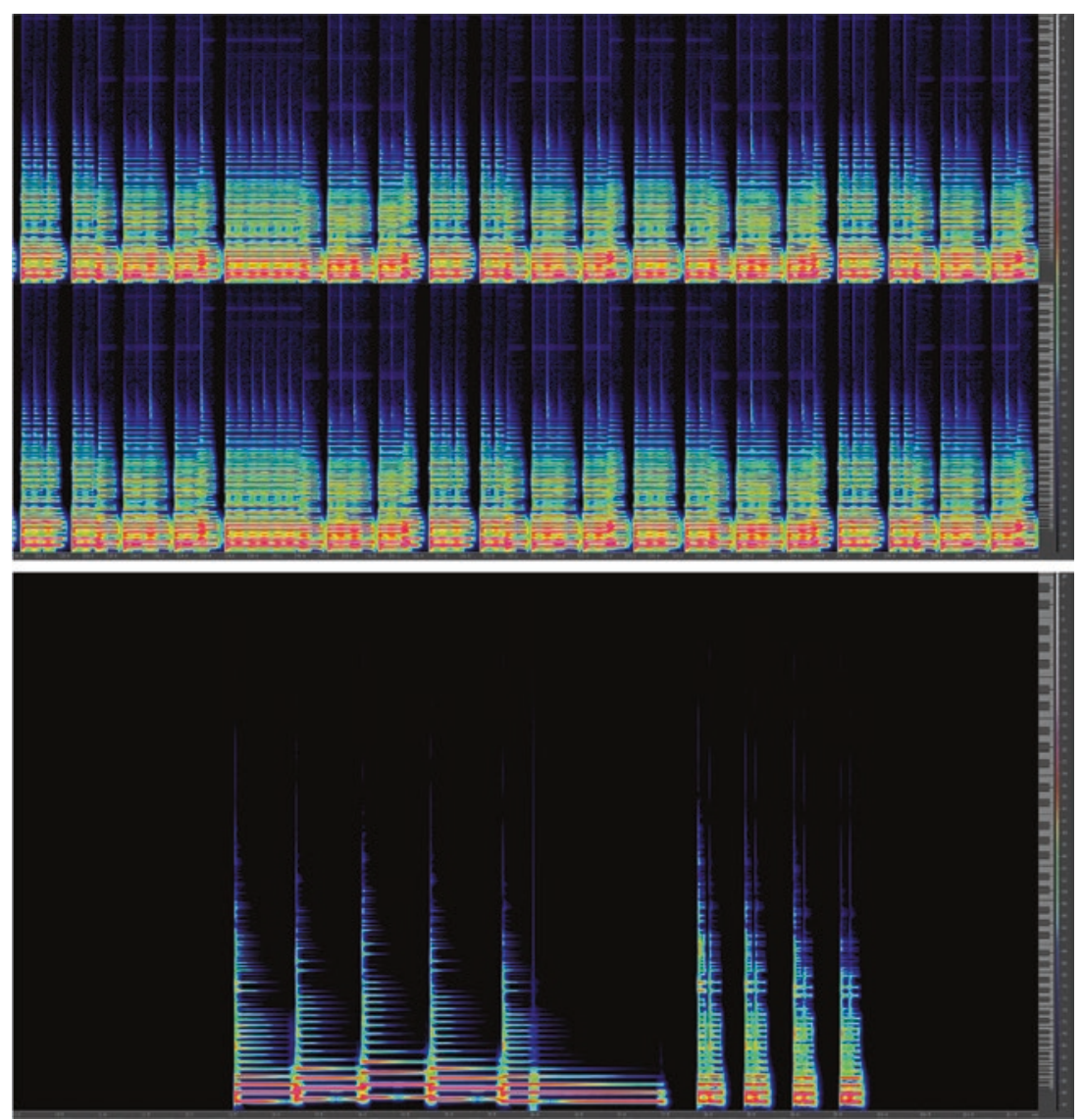

Fig. 11.3 YM2612 channel l (a) and the Yamaha DX7 BASS l preset (b) 
In the B sections of Ice Cap Zone, extra energy is added to the track through channel 4, which plays rapid arpeggios in a harp sound. As with the bass line, the notes and rhythms of the harp signify the new wave genre: rapid arpeggios like these were popular in the 1980s, as they were made much easier to perform due to the availability of computerized arpeggiators. But furthermore, the timbre of the harp is a quintessential FM digital timbre, because FM synthesis was especially praised for its ability to synthesize percussive plucked sounds, like a harp sound, better than other types of synthesizers. Percussive plucked sounds demand the use of inharmonic partials, that is, partials which are not in the typical harmonic, whole-number ratios with the fundamental. Figure 11.4 shows the inharmonicity clearly. The fundamental tone is actually the second-highest horizontal line in this spectrogram, not the lowest; the lowest is like a synthesized "undertone." An undertone creates extra lower resonance without changing the listener's perception of the fundamental pitch. This particular undertone sounds a perfect fourth lower than the fundamental, and is of course not part of the harmonic series, which only naturally proceeds upward from the fundamental.

The synth lead of the B section is not a purely FM sound; while YM channel 2 contributes to the synth lead sound of the B section, PSG channels 1 and 2 also sound in unison with it. By isolating each channel, more details about the composite sound are revealed.

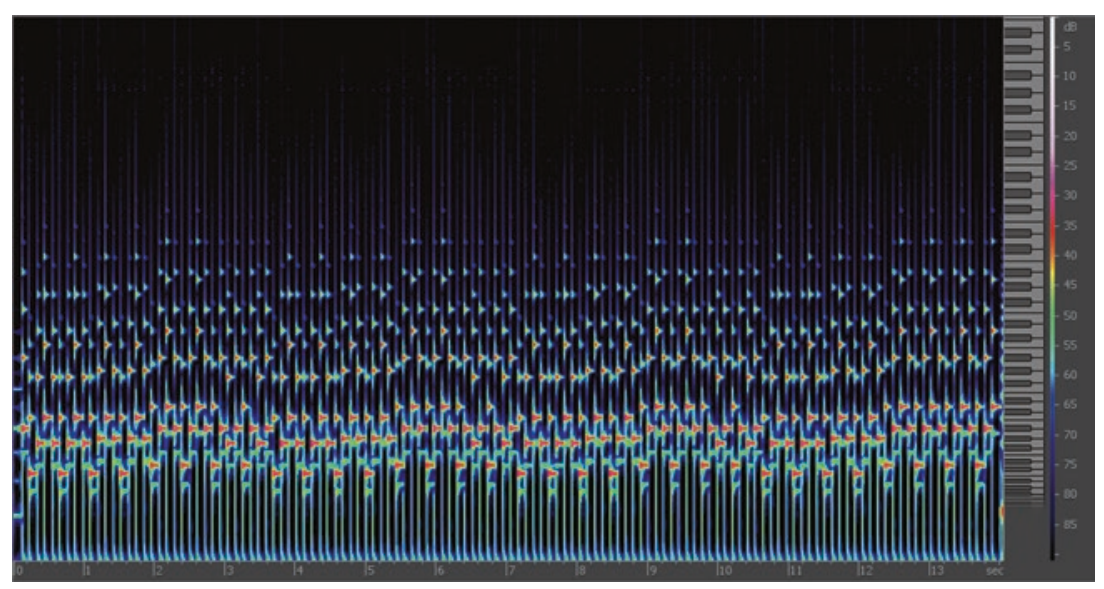

Fig. 11.4 YM channel 4 spectrogram 
Figure 11.5a shows PSG channel 1. The PSG chip generates square waves, which feature only odd-numbered harmonics, in channels 1 through 3. The lack of even-numbered harmonics means that the timbre is somewhat hollow compared to a sound with the full spectrum of harmonics. This hollowness of timbre created through a lack of evennumbered harmonics is a distinguishing timbral feature of the acoustics of the clarinet's sound production; as a result, the PSG sounds slightly clarinet-like. PSG channel 2 exactly duplicates PSG channel 1, except channel 2 is one beat behind channel 1 , and also about 8 to 10 cents flatter. When layered, the two channels create a reverb effect through their displacement from one another, and their varied tunings create slight auditory roughness and beating, which lends a thicker timbre. Figure $11.5 \mathrm{~b}$ is a spectrogram of channels 1 and 2 combined. The strands for each pitch now overlap with one another, visually illustrating the reverb effect. On long notes, the addition of the second channel at a detuned unison is subtly visually apparent, through a very slight thickening of the strand one beat into the tone. Figure $11.5 \mathrm{c}$ shows YM channel 2 during the $\mathrm{B}$ sections. Compared with the PSG channels, the sound is relatively dark, evident through the lack of upper partials. The YM channel uses a wavering tone to create a vibrato effect for this sound, which manifests in the spectrogram as wiggly lines. The dark tone and prominent vibrato effect resembles the tone of a flute.

Combining all three channels together, the clarinet-like hollowness mixes with the flute-like darkness and vibrato to yield a vaguely woodwindsounding synth lead. The spectrographic details of this peculiar blend can be seen in Fig. 11.5d: the odd-numbered partials are strongest, thanks to the square wave PSG channels; the even-numbered partials prominently feature the wavering tone of the YM, creating an ensemble-like timbre.

Although this sound does not lean excessively on FM timbral features, the timbre may still be associated with FM by listeners because of the clarity and precision of the tone-recall the second PSG channel sounding precisely one beat behind the original attack, and the precise 8-to-10-centsflat tuning - and because of its piercing upper-register partials. Clarity in this upper range can be associated with new wave FM synthesizers such as the Yamaha DX7 (Lavengood 2019, 85).

New wave is also associated with the concept of an imagined modernity, which is aesthetically significant in Ice Cap Zone for extramusical reasons. Sonic is snowboarding, not skiing, down the slopes in Ice Cap Zone. Snowboarding was only newly popular in 1994 when Sonic 3 was released; 
a)

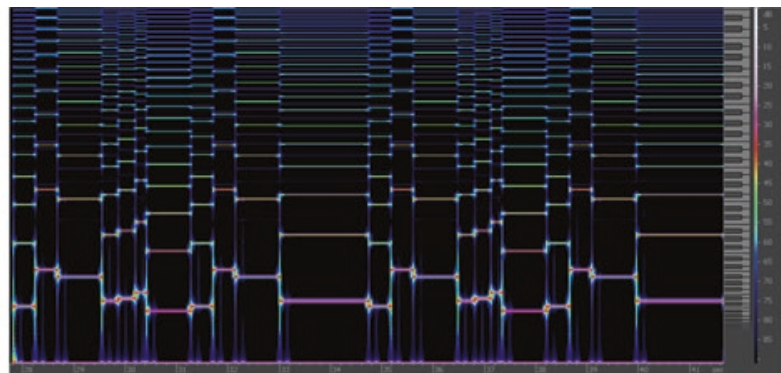

b)

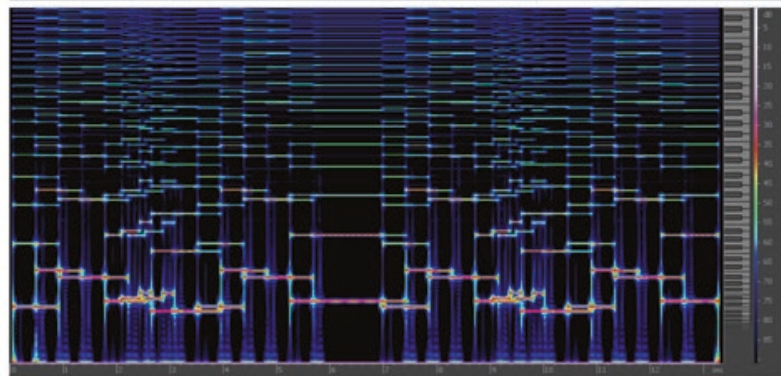

c)

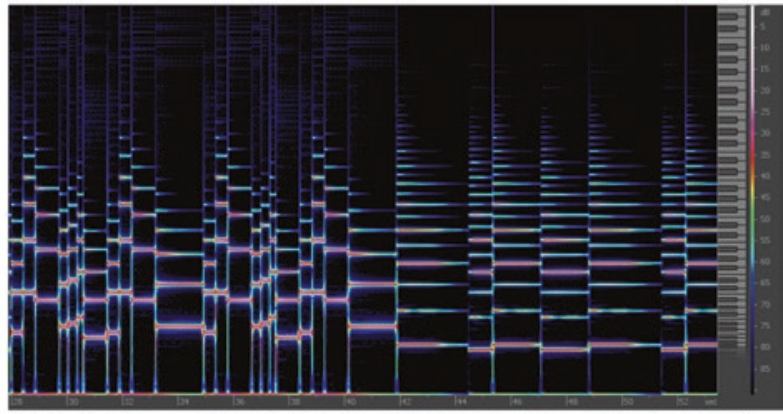

d)

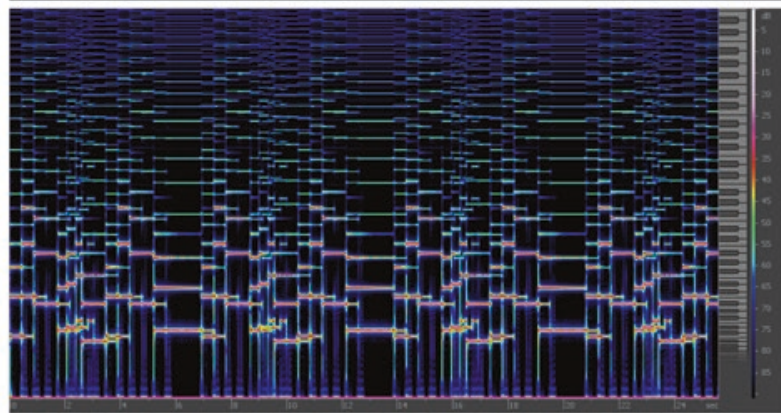

Fig. 11.5 PSG channel l (a), PSG channels 1 and 2 combined (b), YM2612 channel 2 (c), and all three channels combined (d) 
as one indicator of this, snowboarding was not part of the Winter Olympics until 1998 at the Nagano Games. Using this trendy sport shows a rebellious and modern spirit that is culturally associated with new wave music, and of course also with "tough dude" Sonic the Hedgehog.

In short, the new wave genre-topic as used in Ice Cap Zone relates to Ice Cap Zone's setting because the timbres used in this level-especially of the bass, harp, and synth lead-reflect various trends in the new wave genre. The intense, driving rhythms of the bass line and the reliance on arpeggios for texture are quintessentially new wave; the timbral characteristics of the digital instruments overlaps strongly with FM synthesizers that are sonic signifiers of the new wave era. The cultural associations between FM synthesis and the perception of its timbres as being cold and clean add a layer of depth to the player's experience of Ice Cap Zone by sonically reinforcing the visual imagery of the snow.

\section{Marble Garden Zone}

Another surprising pairing of genre and setting is found in Marble Garden Zone. The visual setting appears to be Grecian ruins: white, Doric columns decorated with green and orange designs support platforms made of blue, orange, and white stone blocks mixed together, suggesting various hues of marble; the platforms are covered in what appears to be long, green grass or moss that has grown over the marble platforms. Yet the music sounds Grecian only in the short B sections, while the more dominant A sections evoke the funk genre. In both of these cases, I argue that the genre is not directly reflecting the snowy or Grecian locations, but rather aspects of the contemporary (1992) culture and the narrative behind Sonic as a "real cool" character. Like Ice Cap Zone, Marble Garden Zone does not rely on the stereotypical sounds and styles a composer might first reach for in writing music for its Grecian ruins setting. The form of the track is essentially binary, with an A section that lasts twice as long as the $\mathrm{B}$ section, and a short intro/transition based on A material. This A material, which is sounding $60 \%$ of the time, evokes funk music, articulated through the drum groove, pitch content, and instrumentation. A shuffle funk groove is clearly presented in the drum set and the bass synth. ${ }^{23}$ The melodic material of the A section is based on the C-sharp Dorian scale, and is played by a sound that resembles the electric guitar.

\footnotetext{
${ }^{23}$ Again, transcriptions may be viewed on the author's personal website: www.meganlavengood.com/sonic.
} 
The harmonies alternate between C-sharp minor and F-sharp major, forming a $\mathrm{i}-\mathrm{IV}$ shuttle, a characteristic progression of Dorian-mode pop music and especially funk (Biamonte 2010, 97; Tagg 2011, 177). The harmonies are articulated through accompanying lines whose timbres, to my ear, resemble brass instruments. The "instrumentation" of drum set, bass, brass, and electric guitar thus reinforce my interpretation of this as a funk style.

The only hint of Greekness in Marble Garden Zone arrives in the B section. Here, the funk genre is dropped; excepting the bass synth, which continues in the rhythm presented in the A sections throughout the entire track, the groove and instrumentation changes completely. The key modulates, and a I-iv progression between B major and E minor occurs over a $B$ pedal point in the bass. Rather than using synths that sound like brass and electric guitar, Hammond-organ-like timbres provide the inner voices of the harmony while the melody is carried by an accordion-like sound. To my ear, the accordion is meant to signify "otherness" generally, which then may be related to Greekness specifically, considering both the locale and the pitch content of the melody. ${ }^{24}$ The melody uses both G natural and D sharp prominently, which together form a diminished fourth. This characteristic non-diatonic interval and the upper-neighbor ornamentation create an effect that echoes traditional Mediterranean genres such as rebetika. ${ }^{25}$ The function of this rebetika-esque $\mathrm{B}$ section seems to be essentially matching the style of the music (as indicated through instrumentation and melodic design) to the setting of the level, yet the style is relegated to the $\mathrm{B}$ section, dominated instead by the shuffle-funk A section.

${ }^{24}$ Tagg agrees that the accordion is frequently used to signify otherness in music, citing specifically the French accordion: "The timbre of a musical instrument is often used as part of a genre synecdoche (p. 524 ff.) to connote an 'elsewhere' heard from a musical 'home' perspective, i.e. through the ears of the culture into which it's imported. ... Other wellknown examples of ethnic timbre stereotypes are the French accordion spelling FRANCE (usually PARIS) to the non-French ... countless other examples of ethnic instrument stereotyping will only work if listeners are unaware of the range of moods and functions with which the relevant instrumental sound is associated inside the 'foreign' music culture" $(2012,306)$.

${ }^{25}$ Manuel describes modal music in many Mediterranean cultures. He traces a few continuities among each culture's music, one of which is the frequent use of modes based on the Turkish makam Hicaz (also sometimes spelled Hitzas or Hitzaz). The Greek dromos Hicaz beginning on B would contain the notes B, C, D-sharp, E, F-sharp, G, and A. Pennanen (2008) notes that dromos Hicaz is frequently harmonized with a major tonic chord and a minor subdominant chord $(1989,102)$. Furthermore, Manuel describes characteristic rebetika ornamentation as relying heavily on "hammered-on" upper neighbors (1988, 133). 


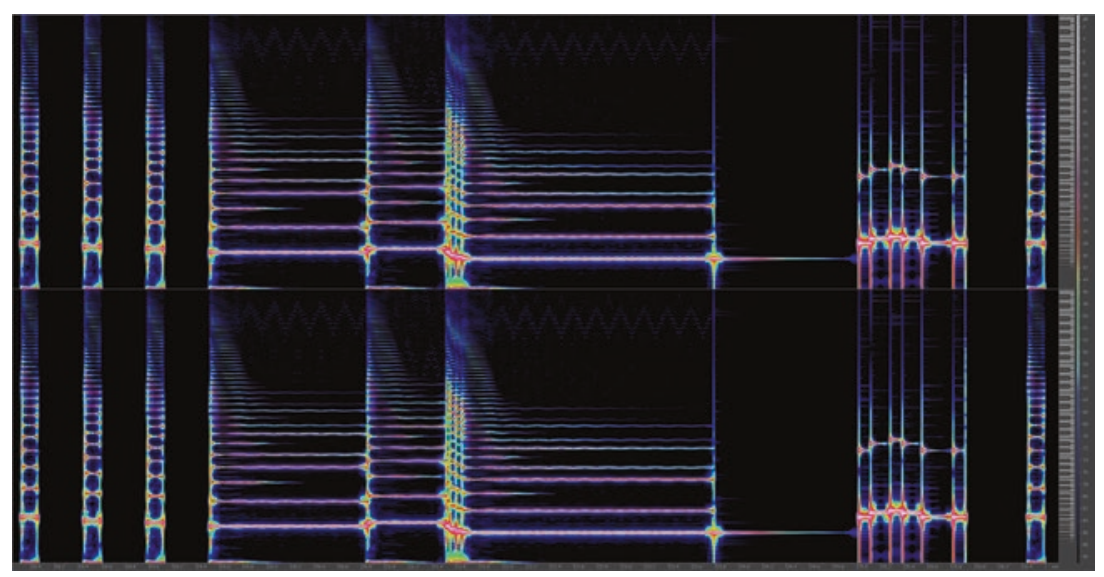

Fig. 11.6 Spectrogram of channel 2 during the A section

The genre of the A section does not necessarily contribute to the perception of a Greek setting for Marble Garden Zone; instead, timbral effects are used to create an aural image of cavernous, marble spaces. This is mostly achieved through the use of delay to create the effect of an echo, similar to what one would hear in a cave, or a bathroom with a lot of tile. Figure 11.6 is a spectrogram of channel 2 during the A section. This is the lead instrument of this track; the timbre is bright (evidenced through the many strong partials even through the upper register) and the attack is sharp. ${ }^{26}$

The YM2612 sound chip cannot generate what one might call "production effects"-reverb, delay, and so on. Yet, Channel 5 in Marble Garden Zone is identical to Channel 2, only displaced by 230 milliseconds. On paper, this may seem like a canon, but the delay is timed to mitigate this effect. At a tempo of 112 beats per minute, each beat is about 535 milliseconds long; thus, a delay of 230 milliseconds means that the second voice comes in at something that looks like half a beat but is noticeably early, by 70 milliseconds. In other words, rather than the lead voice entering on beat 1 and the following voice on beat 1.5 , the following voice enters on beat 1.42. The timing is precise like a canon, yet its lack of

\footnotetext{
${ }^{26}$ To my ear, this resembles the electric guitar I mentioned previously, but the resemblance is likely too subtle to responsibly and definitively say this synthesized sound is meant to sound like a guitar.
} 
clear placement on the metrical grid allows it to be experienced as an echo effect rather than as a double attack or as eighth note subdivisions. The echo is more prominent because of this timing too: Bobby Owsinski, in his book The Mixing Engineer's Handbook, states this simply in two section headers: "If Delays Are Not Timed to the Tempo of the Track, They Stick Out" and conversely, "Reverbs Work Better When They're Timed to the Tempo of the Track" $(2013,86) .{ }^{27}$ The echo effect sonically engineers the illusion of a deeper, three-dimensional space. ${ }^{28}$ Generally speaking, funk music is not typically produced with noticeable reverb or delay in its melodic lines. ${ }^{29}$ The subversion of generic expectations creates a special significance in what might seem to be a simple production effect (echo).

The use of reverb provides one connection between the quasi-Grecian setting and the funk style of the A sections, but-especially because funk doesn't typically use extensive and noticeable reverb as in the echo effect here-one wonders still what the purpose is of evoking the funk genre at all, given that its origins in Black American culture seems to be quite at odds with (an outsider's perception of) the culture of Greece. Yet this opposition between Greece and funk itself generates a kind of narrative about Sonic. In analyzing the music of Ice Cap Zone, I argued that new wave music was meant to sound explicitly modern. Similarly, I interpret the funk-style music in Marble Garden Zone to be a use of a genre-topic that is conspicuously incongruous with the physical locale, and that in turn draws attention to Sonic's status as a contemporary figure (that is, contemporary to 1992) and as an outsider or visitor. As one concrete example of this genre opposition, consider the harmonic languages used in the A and B sections, which serve as synecdoches for these opposing genre-topics. While the A sections use the Dorian chord shuttle between i and IV, the B sections invert the chord quality, using a major I chord and

\footnotetext{
${ }^{27}$ Note that while reverb and delay are separate effects, Owsinski conflates them here because they are both related to timing.

${ }^{28}$ Recording engineers and producers are well aware of this fact. See, for example, Owsinski $(2013,77)$ : "The fourth element of a mix is dimension, which is the ambient field where the track or tracks sit. Dimension can be captured while recording but usually has to be created or enhanced when mixing by adding effects such as reverb, delay, or any of the modulated delays such as chorusing or flanging."

${ }^{29}$ By "noticeable," I mean delay that is noticed as such by a casual, untrained listener. A recording engineer or a musician well-versed in production techniques would likely notice even subtle reverb/delay that is meant for "fattening up" a sound; however, the reverb/ delay in this case is probably not intended to be heard by a casual listener.
} 
a minor iv chord, thus generating a non-diatonic collection as the implied basis for the tonal content of the B section. The stylistic opposition is further illustrated through timbral effects, namely the use of timing to create an echo effect, and contrasting instrumentations as genre synecdoches for each of their respective genres. The stylistic oppositions thus are representative of Sonic as the player's contemporary protagonist in an Othered world of Greek antiquity.

\section{CONCLUSION}

Exhaustively listing all components of music (and outside of music) that constitute a genre is an impossible task for many reasons, not the least of which is the constantly shifting boundaries of any given genre, but there seems to be some consensus about the great significance of timbre in genre identification (Leydon 2010; Gjerdingen and Perrott 2008; Blake 2012; Wallmark 2014). While many excellent studies have essentially correlated instrumentation with timbre in their discussions of genre and style, as in Tagg's concept of the instrument as a genre synecdoche, video game music can obfuscate the idea of instrumentation altogether. When instrumentation alone cannot function as a genre indicator, timbre provides many aural cues that direct listeners to a specific extramusical context. In other words, acoustic features of sounds, observable through spectrograms, can be the triggers for these larger, intertextual associations.

Discussion of meaning in video game music, or any repertoire, benefits from a consideration of what the function or purpose of that music is. In video games, as in film, music can play a variety of roles, from simplistic to complex. In the postmodern society of 1992, a composer of video game music can reasonably assume that listeners are familiar with many genres, and thus bring their own preconceptions about what that genre means to their gameplay experience. Thus, in one way or another, the presence of a given genre-topic in the game soundtrack functions to enhance immersion in the gameplay by creating a multi-sensory and intertextual experience for the player that goes beyond pressing buttons on a controller and watching visuals on a screen, and extends into the player's memory and personal experience with that genre. This relationship could be a simplistic one, like using carnival music for a carnival-themed level, but even when the function of the chosen musical style is not obvious, as in Ice Cap Zone and Marble Garden Zone, the listener is still consciously or subconsciously relating the gameplay to the style of music, and the style to the broader 
social contexts of that genre. I find that the obvious style-setting pairings make the surprising pairings seem more intentional-if the composers indeed do sometimes rely on a simple style-setting pairing, what is communicated when a composer instead flouts these more obvious pairings? Even though Sonic 3 is a game with very minimal storyline and character development, its narrative is still palpable in the subversive pairings of new wave and funk music with snowy and Grecian settings, respectively. Musical genres, styles, and topics can be represented partially through musical timbres, and subversion of generic or stylistic expectations can serve to communicate subtle and complex ideas about the narrative of the game.

\section{REFERENCES}

Bannister, Richard. 2016. Software: Audio Overload. Personal website. http:// www.bannister.org/software/ao.htm.

Biamonte, Nicole. 2010. Triadic Modal and Pentatonic Patterns in Rock Music. Music Theory Spectrum 32 (2): 95-110. https://doi.org/10.1525/ mts.2010.32.2.95.

Black o White. 2009. Brad Buxer: Musicien, Arrangeur.

Blake, David K. 2012. Timbre as Differentiation in Indie Music. Music Theory Online 18 (2). http://mtosmt.org/issues/mto.12.18.2/mto.12.18.2.blake.php.

Boone, Christine. 2018. Gendered Power Relationships in Mashups. Music Theory Online 24 (1). http://mtosmt.org/issues/mto.18.24.1/mto.18.24.1.boone.html.

Brackett, David. 2016. Categorizing Sound: Genre and Twentieth-Century Popular Music. Oakland: University of California Press.

Cateforis, Theo. 2011. Are We Not New Wave? Modern Pop at the Turn of the 1980s. Ann Arbor: University of Michigan Press.

Collins, Karen. 2008. Insert Quarter to Continue: 16-Bit and the Death of the Arcade. In Game Sound: An Introduction to the History, Theory, and Practice of Video Game Music and Sound Design, 37-62. Cambridge, MA: MIT Press.

Elen, Richard. 1986. Music Technology \& the Alan Parsons Project. Sound on Sound, May.

Gjerdingen, Robert O., and David Perrott. 2008. Scanning the Dial: The Rapid Recognition of Music Genres. Journal of New Music Research 37 (2): 93-100.

HXC. 2005. Roger Hector: Director of STI Interviews. Secrets of Sonic Team, October. http://sost.emulationzone.org/sonic_3/interview/rogerhector.htm.

Johnson, Thomas. 2017. Tonality as Topic: Opening A World of Analysis for Early Twentieth-Century Modernist Music. Music Theory Online 23 (4). https:// doi.org/10.30535/mto.23.4.7. 
Johnson, Thomas. 2018. A Music-Theoretical Approach to Genre in PostMillennial Popular Music. PhD diss., City University of New York.

Lavengood, Megan. 2019. "What Makes It Sound '80s?": The Yamaha DX7 Electric Piano Sound.” Journal of Popular Music Studies 31 (3): 73-94. https:// doi.org/10.1525/jpms.2019.313009.

Leydon, Rebecca. 2010. Recombinant Style Topics: The Past and Future of Sampling. In Sounding Out Pop: Analytical Essays in Popular Music, ed. Mark Spicer and John Covach, 193-213. Ann Arbor: University of Michigan Press.

Lynch, Joe. 2016. Michael Jackson Wrote 'Sonic the Hedgehog 3' Music: Crazy Theory Confirmed? Billboard, January 26. https://www.billboard.com/articles/news/6858197/michael-jackson-sonic-hedgehog-3-music-theory.

Manuel, Peter. 1988. Popular Musics of the Non-Western World: An Introductory Survey. Oxford University Press.

- 1989. Modal Harmony in Andalusian, Eastern European, and Turkish Syncretic Musics. Yearbook for Traditional Music 21: 70-94.

“Michael Jackson Involvement with Sonic 3.” 2017. Wiki. Sonic Retro, September 22.http://info.sonicretro.org/Game_Development:Sonic_the_Hedgehog_3/ Music.

Mirka, Danuta. 2014. Introduction. In The Oxford Handbook of Topic Theory, ed. Danuta Mirka, 1-57. New York: Oxford University Press.

Moore, Allan F. 2001. Rock: The Primary Text. 2nd ed. Brookfield: Routledge.

Owsinski, Bobby. 2013. The Mixing Engineer's Handbook. 3rd ed. Boston: Cengage Learning PTR.

Pennanen, Risto Pekka. 2008. The Development of Chordal Harmony in Greek Rebetika and Laika Music, 1930s to 1960s. British Journal of Ethnomusicology 6 (1): 65-116.

Ratner, Leonard G. 1980. Classic Music: Expression, Form and Style. New York: Schirmer Books.

Schloss, Joseph G. 2004. Making Beats: The Art of Sample-Based Hip-Hop. Middletown, CT: Wesleyan University Press.

Sega Enterprises, Ltd. 1991. Sonic the Hedgehog Instruction Manual.

Tagg, Philip. 2011. Everyday Tonality: Towards a Tonal Theory of What Most People Hear. Edition 1.3. New York: The Mass Media Music Scholars' Press.

- 2012. Music's Meanings: A Modern Musicology for Non-Musos. Edition 2.4.2. New York: The Mass Media Music Scholars' Press.

Vail, Mark. 2005. A Conversation with Bob Moog: Analog vs. Digital Sound Generation. Keyboard, January.

Van Luling, Todd. 2016. The Michael Jackson Video Game Conspiracy. The Huffington Post, January 25. http://testkitchen.huffingtonpost.com/ michaeljacksonsonic/. 
Wallmark, Zachary Thomas. 2014. Appraising Timbre: Embodiment and Affect at the Threshold of Music and Noise. PhD diss., University of California Los Angeles.

Weinstein, Deena. 2000. Heavy Metal: The Music and Its Culture. Rev. ed. New York: Da Capo Press.

Williams, Justin A. 2013. Rhymin' and Stealin': Musical Borrowing in Hip-Hop. Ann Arbor: University of Michigan Press. 